\section{Hyposensibilisierung steigert die Lebensqualität bei allergischer Rhinitis}

\author{
Bei Patienten mit einer ganzjährigen allergischen Erkrankung \\ der oberen Atemwege kann eine spezifische Immuntherapie die \\ Lebensqualität spürbar steigern.
}

$\mathrm{P}$ atienten mit perennialer Rhintis allergica fühlen sich in ihrer Lebensqualität mindestens ebenso beeinträchtigt wie solche mit mildem bis mäßigem Asthma. Daher stellten amerikanische Allergologen diesen Aspekt ganz in den Vordergrund einer Evaluation des Nutzens der spezifischen Immuntherapie bei 25 erwachsenen Patienten mit allergischer Rhinitis: Mit Hilfe eines standardisierten Fragebogens versuchte man die Lebensqualität von Patienten unter medikamentöser Therapie zu objektivieren und stellte die Werte denjenigen unter zusätzlicher spezifischer Immuntherapie gegenüber. Gleichzeitig wurden die entsprechenden Kosten verglichen.

In die Ermittlung der Lebensqualität gingen allgemeine sowie krankheitsspezifische Items ein. Sowohl Behandlungskosten als auch der Lebensqualität-Score wurden jeweils für das Jahr 0 (vor Beginn der Hyposensibilisierung) und den Verlauf der Behandlung (Jahre 1, 2 und 3) ermittelt.

Die Patienten beurteilten ihre Beschwerden anhand einer Skala von 0 (keine Probleme) bis 6 (extrem quälend). Der Lebensqualitäts-Index, ein Gesamtwert, der aus sämtlichen erfaßten Symptom-Scores kalkuliert wurde (der also invers mit dem Komplex Lebensqualität korreliert), ging im ersten Jahr der Behandlung mit Allergenextrakt merklich $(p<0,001)$ zurück. Im zweiten Hyposensibilisierungs-Jahr erfuhren die Behandelten eine weitere signifikante umfassende Erleichterung - die ermittelten Daten stehen für einen geringeren Ausprägungsgrad der Symptome und substantiell besseres Befinden. (Für das 3. Jahr Immuntherapie waren die Angaben nicht komplett.)

In die Summe der Kosten gingen die ärztliche Untersuchung, Hauttests,
Medikamente und die spezifische Immuntherapie ein, also der Allergenextrakt plus Verabreichen der Injektionen. Die Hyposensibilisierung erhöhte die Gesamtaufwendungen im ersten Jahr. Im Laufe der 3jährigen Behandlung waren die Auslagen für die Pharmakotherapie jedoch stark rückläufig - ein Trend, der zwischen den Jahren 3 und 1 signifikant war, und den ursprünglichen Anstieg kompensierte.

Die Botschaft der Studie: Die spezifische Immuntherapie ist bei ausge-
B ei saisonaler Rhinitis ist unter fortgesetzter Allergen-Exposition eine allergische Entzündung auch während der symptomfreien Intervalle nachweisbar. Daher sollten Medikamente mit antiallergischen Eigenschaften während der gesamten Dauer der Allergenexposition gegeben werden.

Cetirizin reduziert zusätzlich zu seiner hochselektiven antihistaminischen Aktivität die Eosinophilen- und Neutrophilen-Infiltration. In einer randomisierten Doppelblindstudie erhielten 20 Patienten mit saisonaler allergischer Rhinokonjunktivitis während der Pollensaison randomisiert entweder täglich eine Tablette $(10 \mathrm{mg})$ Cetirizin oder Plazebo. Zusätzlich konnte je nach Bedarf pro Tag eine weitere Cetirizin-Tablette eingenommen werden, wovon die Plazebo-Patienten erwartungsgemäß häufiger Gebrauch machten. wählten Patienten geeignet, die Lebensqualität von Patienten mit Rhinitis allergica im Zusammenspiel mit den bewährten Strategien Allergenkarenz - so weit möglich - und Pharmakotherapie spürbar zu verbessern. Dieser Vorteil scheint sich zudem auf mittlere Sicht nicht in höheren Kosten niederzuschlagen.

Der Pferdefuß der Untersuchung: Die Studie wurde zwar, insbesondere was Auswahl der Patienten und Ausschöpfung von Sanierungsmaßnahmen angeht, sorgfältig durchgeführt, es handelt sich jedoch um retrospektive Auswertungen. Die Autoren räumen daher ein, daß eine prospektive Studie mit zwei Therapiearmen bei gut vergleichbaren Kollektiven angezeigt wäre, um die Resultate zu bestätigen.

(wpa)

Quelle: Kumar P, Kamboj S. et al.: The cost of care and quality of life in patients with allergic rhinitis on allergen immunotherapy. Allergy Clin. Immunol. Int. (1997); 9: 133-35.

\title{
Allergische Rhinitis:" Bedarfsorientierte Medikation verschenkt Therapiepotential
}

\author{
Patienten mit allergischer Rhinitis profitieren von einer \\ kontinuierlichen Behandlung mit einem $\mathrm{H}_{1}$-Blocker mehr, als wenn \\ sie das Medikament nur nach Bedarf einnehmen.
}

In beiden Gruppen nahmen die Beschwerden in der 2. Behandlungswoche signifikant ab. In der 3. und 4. Woche gingen die Symptome unter der kontinuierlichen Cetirizin-Gabe deutlich stärker zurück als unter Plazebo pus bedarfsorientierter Cetirizin-Einnahme. Die massive Infiltration der Nasenschleimhaut mit Entzündungszellen wurde in beiden Gruppen deutlich reduziert, die Abnahme war aber unter der kontinuierlichen Therapie signifikant stärker.

Die kontinuierliche Einnahme des Antihistaminikums während der Pollensaison erwies sich der unregelmäßigen Einnahme des Medikaments in Abhängigkeit von den akuten $\mathrm{Be}$ schwerden damit als überlegen. (wpa)

Quelle: Ciprandi G, Passalacqua G, et al.: Continuous versus on demand treatment with cetirizine for allergic rhinitis. Ann. Allergy Asthma Immunol. (1997); 79: 507-11. 